\title{
Platelet-vessel wall interaction in normal and diabetic rats. Quantification using scanning electron microscopy and in vivo recording of thrombus formation
}

\author{
R. H. BOURGAIN*, J. X. WU**, R. ANDRIES* and L. MAES* \\ * Vrije Universiteit Brussel \\ Faculty of Medicine \\ Laboratory of Physiology and Physiopathology \\ Laarbeeklaan 103 \\ B-1090 Brussels, Belgium \\ ** Vrije Universiteit Brussel \\ Academic Hospital \\ Department of Pathology \\ Laarbeeklaan 101 \\ B-1090 Brussels, Belgium
}

Key words: experimental diabetes, thrombosis, morphology, arachidonate cascade

\section{Summary}

The morphology of the mesenteric arterial wall, and the role of the arachidonate cascade in ADP-induced thromboformation, is studied in two groups of streptozotocin-induced diabetic rats, in comparison to a group of normal rats.

Light microscopy demonstrates the dilated state of the arteries of the diabetic animals, together with the diminished wall thickness in "young diabetics", and an increased thickness in "old diabetics", as compared to normal animals. Scanning electron microscopy shows the area of local deendothelialization in the arteries of "young diabetics" to be larger than in both other groups.

Combined use of a cyclooxygenase and a prostacyclin synthetase inhibitor, reveals that the decreased $\mathrm{PGI}_{2}$ release from arteries of diabetic animals, can be related to a diminished activity of the prostacyclin synthetase.

\section{Introduction}

It is well known that diabetic patients frequently develop vascular complications involving an enhanced propensity to arterial thrombosis and atherogenesis ${ }^{1,2)}$. According to Ross and Glomset ${ }^{3)}$, the risk factors triggering or accelerating atherogenesis comprise endothelial damage leading to local platelet adhesion, aggregation and thrombosis. These mechanisms precisely could well be enhanced in diabetic patients ${ }^{4}$ and result from modifications

* Dr. Wu, J.X. is a visiting scientist from Bethune Medical College, The People's Republic of China 
in the arachidonate cascade as indeed it has been demonstrated that in diabetes mellitus the production of thromboxane $\mathrm{A}_{2}$ is significantly increased $^{5)}$ while the endothelial cells generate less prostacyclin ${ }^{6,7)}$. Conflicting data have been published ${ }^{8-11}$ in this particular domain and therefore closer scrutiny is required.

In a previous publication ${ }^{12)}$, an in vivo method for the induction and registration of arterial thrombi was described in detail. It was clearly established that the arachidonate cascade is deeply involved in plateletvessel wall interaction which ultimately leads to thrombogenesis ${ }^{13)}$. Transmission and scanning electron microscopic data ${ }^{14) 15}$ demonstrate the type of lesions involved such as local endothelial cell detachment, platelet adhesion and thrombus formation.

In order to further investigate platelet-vessel wall interaction as well as the alterations occurring in the vessel wall itself, thromboformation was induced in diabetic rats. The morphology of the arterial wall and the aspect of thrombus formation in relation to the arachidonate cascade was assessed and compared in a group of normal and diabetic animals.

\section{Materials and Methods}

Twenty-seven male white Wistar rats were used. Diabetes was induced by the intravenous injection of sterptozotocin $(65 \mathrm{mg} / \mathrm{kg}$ in $0.1 \mathrm{M}$ citrate buffer). Seven were injected at a weight of 130 to $160 \mathrm{~g}$, and the experiments performed six weeks later (young diabetics). Thirteen animals were injected at a body weight of 250 to $300 \mathrm{~g}$ and examined 2.5 to 3 months later (old diabetics); seven animals served as controls. All rats were checked for glucosuria, daily consumption of water and diuresis; at no time insulin is administered. Three animals of the control group and of the group of "old diabetics" were chosen randomly for analysis of insulin, glucose and phospholipid concentrations in the blood.

\section{Tissue preparation for ultrastructural control}

For scanning electron microscopy (SEM), and eventually for light microscopy (LM), the rats were perfusion fixed at physiological pressure ${ }^{15)}$ by $2 \%$ glutaraldehyde in $0.1 \mathrm{M}$ cacodylate buffer. For SEM examination, the arterial segments were postfixed in $1 \% \mathrm{OsO}_{4}$ for one hour, dehydrated in increasing concentrations of ethanol and critical point dried. The vessels were glued onto special specimen mounts, opened longitudinally as described by Reidy and Schwartz ${ }^{16}$ and coated with 10-15 nm of gold. For LM, the vessels were stained with $2 \%$ uranyl acetate in veronal buffer following postfixation with $\mathrm{OsO}_{4}$, dehydrated and stained with toluidine blue for diameter and wall thickness measurements.

Induction and registration of the thrombus

Technical details concerning the operative procedure, induction of local de-endothelialization and thrombus generation, as well as the technique for registration have been described in detail ${ }^{12)}$. Briefly summerized, the method consists in the dissection over a distance of 2-3 $\mathrm{mm}$ of a branch of the mesenteric artery following gentle extraction of a loop of small intestine. The animal is intubated and artificially ventilated all through the experiment, while heart rate and body temperature are continuously monitored. 


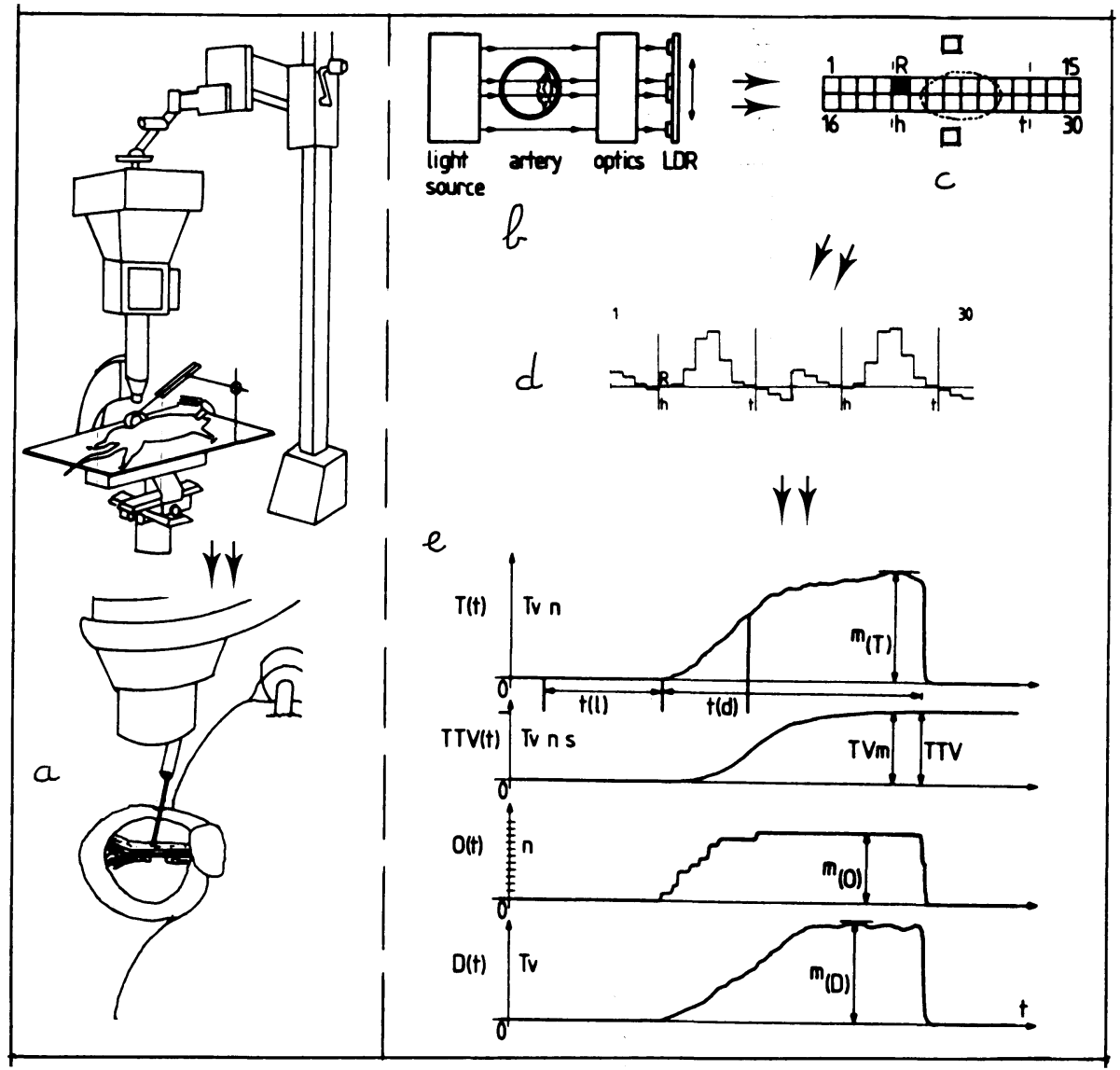

Fig. 1 Opto-electronic device for the detection and registration of thrombotic parameters in an arterial segment in vivo.

The left part of the figure demonstrates the general set-up. The mesenteric preparation is continuously superfused (a) by Ringer solution $\left(37^{\circ} \mathrm{C}-1 \mathrm{ml} / \mathrm{min}\right)$. The dissected arterial segment is projected (b) in a dark room unit onto a detection device via a fixed microscope. The detection device $(c)$ consists of a matrix $(2 \times 15)$ of light depending resistances (LDR), which convert the changes in light intensity into potential variations which are monitored on oscilloscope (d). The right bottom part represents the thrombus parameters of an ADP-induced thrombus (e), computed from the variations in electrical potentials.

Through transillumination by means of a cold light source, the image of the investigated arterial segment is projected in dark room onto a set of thirty light depending resistances (LDR) arranged in two columns of fifteen elements each (Fig. 1). An LDR has the property to change its internal resistance in function of variations in light intensity, which occur when a platelet thrombus appears in the arterial lumen, and these variations will be recorded as changes in electrical potentials and registered as such via a multiplexing device.

In the present investigation four discriminating parameters are computed: - the $m_{(T)}$ parameter, which represents the maximal value on the $\mathrm{T}(\mathrm{t})$ 
Table 1 Outer diameter, wall thickness and respective ratio of mesenteric arteries of normal and diabetic rats.

\begin{tabular}{|l|rrrrr|l|}
\hline & $\begin{array}{c}\text { Young } \\
\text { diabetics }\end{array}$ & Sign. & Normals & Sign. & $\begin{array}{c}\text { Old } \\
\text { diabetics }\end{array}$ & \\
\hline Diameter & 367.2 & $\mathrm{p}<0.05$ & 286.2 & $\mathrm{p}<0.001$ & 398.6 & means \\
(a) $-\mu \mathrm{m}$ & 10.6 & & 5.8 & & 16.6 & sem \\
Thickness & 19.7 & $\mathrm{p}<0.05$ & 25.4 & $\mathrm{p}<0.001$ & 35.1 & means \\
(b) $-\mu \mathrm{m}$ & 1.0 & & 0.6 & & 1.5 & sem \\
Ratio & 5.3 & $\mathrm{p}<0.001$ & 9.1 & $\mathrm{n} . \mathrm{s}$. & 8.8 & means \\
(b/a)-\% & 0.2 & & 0.2 & & 0.2 & sem \\
\hline
\end{tabular}

The represented values are the means \pm standard errors of the means, from 32 arterial segments of 3 normal rats, from 24 segments of 2 "young", and from 20 segments of 2 "old diabetic" rats.

Significance levels are obtained via Student's t-test for unpaired data.

Significance levels between "young" and "old diabetic" animals are

for diameter: n.s.

for thickness: $\mathrm{p}<0.01$

for ratio: $\mathrm{p}<0.001$

curve. This curve is obtained from the sum of all electrical potentials registered on the LDR elements covered by the image of the thrombus during each time interval of thirty milliseconds.

- the $m_{(D)}$ parameter, which is the maximal value on the $\mathrm{D}(\mathrm{t})$ curve. This curve represents at each time interval the highest potential deviation on one of the LDRs covered by the projected image of the thrombus.

- the TTV parameter, representing the maximal value of the TTV(t) curve, which is the time integrated curve of the $T(t)$ curve.

- the TVM parameter which is the value of the TTV(t) curve up to the point where $m_{(T)}$ is reached.

Aspirin (ASA) at $10^{-2} \mathrm{M}$ in topical superfusion is used as an inhibitor of cyclooxygenase activity. Tranylcypromine at $2 \times 10^{-3} \mathrm{M}$ is used for the inhibition of prostacyclin synthetase.

\section{Results}

Out of twenty diabetic animals, four died before the end of the experiments; the six remaining "young diabetic" ones weighted only $7 \%$ less than normal control rats of the same age, but in the "old diabetic" group, the ten surviving animals had a mean body weight reduced by $41 \%$ as compared to the control group. In these animals, water consumption and urinary output increased very markedly and all presented positive glucosuria. The glucose concentration increased from $189.3 \mathrm{mg} \%$ (range 181-221) to $616.5 \mathrm{mg} \%$ (range 515-735), the phospholipid concentration from $89.5 \mathrm{mg} \%$ (range 43-107) to $155.6 \mathrm{mg} \%$ (range 100-233), while insulin decreased from $98.3 \mathrm{mU} / 1$ (range 5-208) to $11.5 \mathrm{mU} / 1$ (range 9-14). The concentrations of these substances were determined only in the latter group, because the experiments with ASA and tranylcypromine were compared to those performed in normal rats of the same age.

Diameter of the artery and wall thickness

In Table 1 are represented the results of LM measurements of the outer 

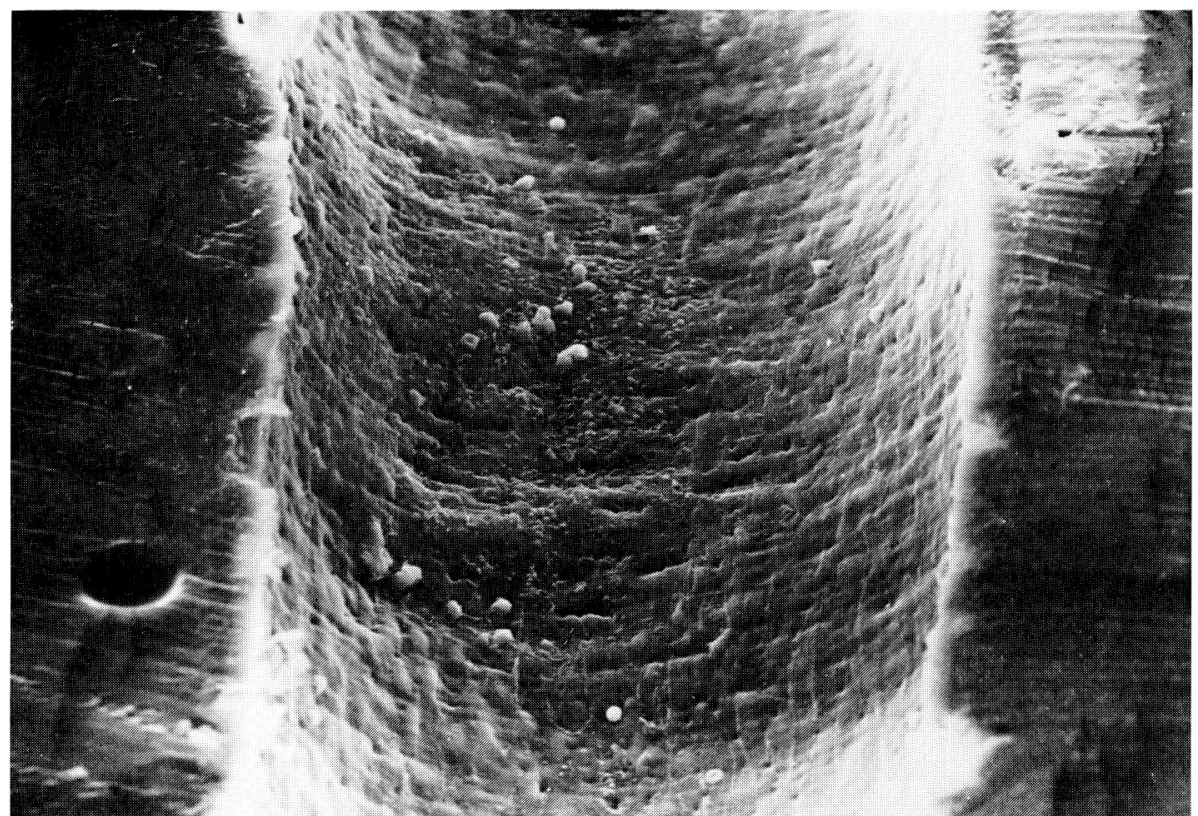

Fig. 2 SEM of a branch of the mesenteric artery of a normal rat, following electrical de-endothelialization $(\times 300)$.

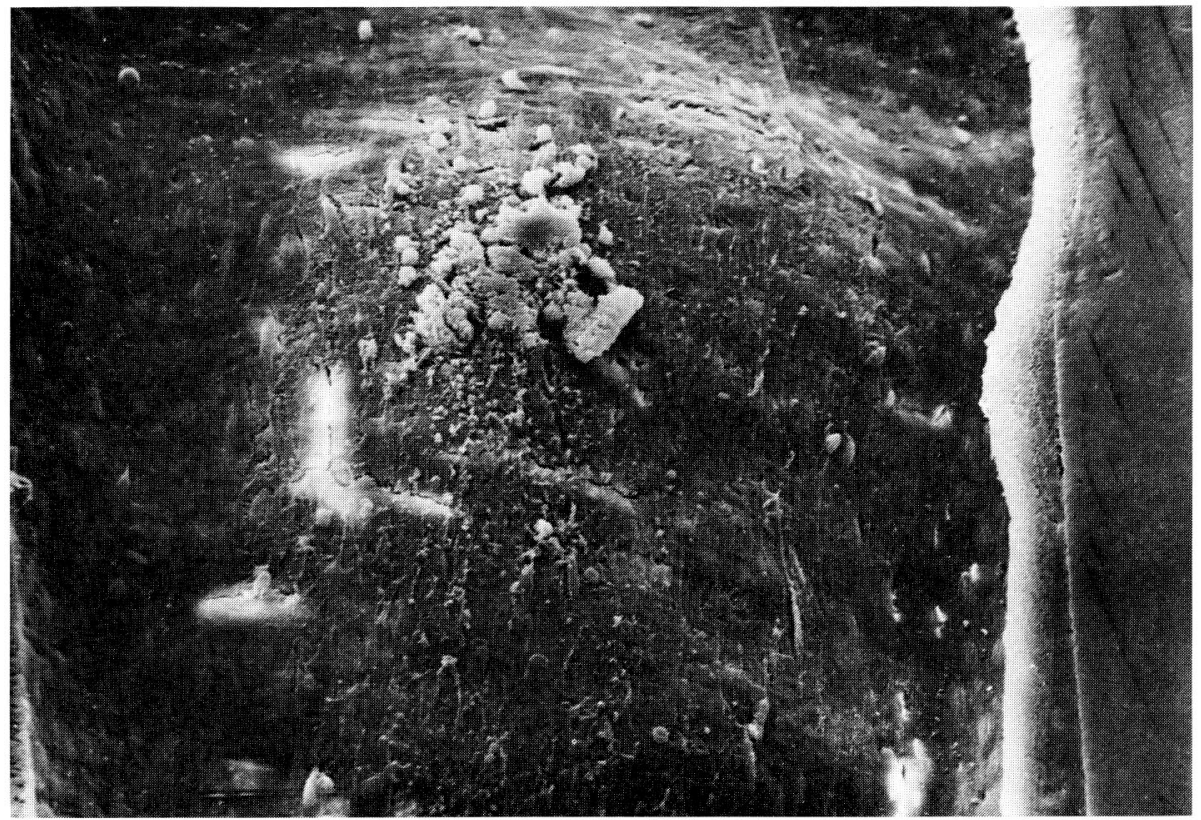

Fig. 3 SEM of a branch of the mesenteric artery of a "young diabetic" rat, showing a large de-endothelialized area and a small thrombus $(\times 300)$.

diameter, the wall thickness and the respective ratio's of the mesenteric arteries of normal and diabetic rats. Both diameter and thickness of "old diabetic" arteries are significantly increased as compared to normal vessels; 


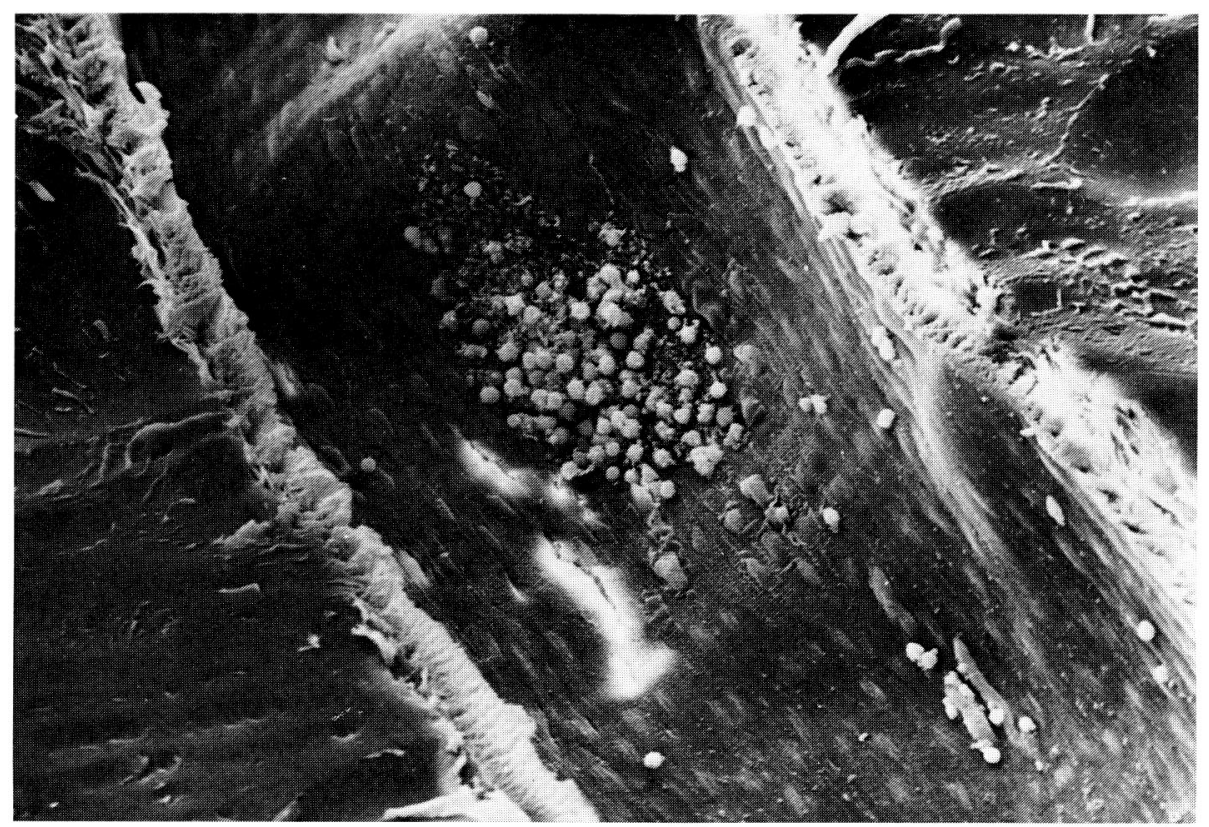

Fig. 4 SEM of the de-endothelialized area in a branch of the mesenteric artery of an "old diabetic" rat, comparable to a lesion in a normal rat, which is covered by leukocytes $(\times 300)$.

this results in a non-significant difference in ratio values. The opposite alterations in diameter and thickness of "young diabetic" arteries, result in a highly significant difference in ratio as compared to normal arteries. The increase in vessel diameters of both diabetic groups is statistically indistinguishable.

\section{Induction of the lesion and ultrastructural control (SEM)}

Local de-endothelialization is induced by application of a small electrical current ( 1 minute-inversing polarity every 5 seconds) through a platinum electrode applied onto the dissected arterial segment. The intensity is related to the diameter of the artery by the equation:

current $(\mu \mathrm{A})=$ diameter $(\mu \mathrm{m}) \times 0.04$

The diameter is approximated through a micrometer device adapted to an operation microscope. Three thrombi are induced in succession by the topical superfusion of an ADP solution; the animal is then immediately perfusion fixed.

In normal rats (Fig. 2), the current application results in a desquamation of 20 to 30 adjoining endothelial cells; the surrounding cells show signs of retraction and flap formation, their nuclei bulging out very clearly into the bloodstream. In the "young diabetics" (Fig. 3), the lesion appears to be larger than in normal animals; more platelets stick to the deendothelialized area and they form a very small thrombus which does not embolize. In the "old diabetic" group (Fig. 4), the lesion shows much resemblance to the one induced in normal rats. The white blood cells adhering to the site of desquamation, are mainly polymorphonuclears in normal rats, but in diabetic animals monocytes are more frequently found, 
Table 2 Effect of continuous ASA superfusion on thromboformation induced by ADP in normal rats.

\begin{tabular}{|c|c|c|c|c|c|c|c|}
\hline Parameter & 10 & 22 & $\begin{array}{c}\text { Superfusion } \\
34\end{array}$ & $\underset{46}{\text { (minutes) }}$ & 58 & 70 & \\
\hline \multirow[t]{2}{*}{$m(T)$} & 53.7 & 55.0 & 76.6 & 95.2 & 101.9 & 110.8 & means \\
\hline & 9.0 & 13.6 & 20.0 & 22.5 & 22.3 & 23.1 & sem \\
\hline \multirow{2}{*}{$m_{(D)}$} & 60.2 & 70.8 & 82.6 & 102.1 & 94.2 & 101.4 & means \\
\hline & 6.0 & 16.0 & 18.6 & 19.8 & 17.9 & 20.1 & sem \\
\hline \multirow[t]{2}{*}{$T V M$} & 31.7 & 55.8 & 68.2 & 109.2 & 103.9 & 109.4 & means \\
\hline & 4.3 & 22.8 & 24.1 & 45.6 & 42.8 & 45.8 & sem \\
\hline \multirow[t]{2}{*}{$T T V$} & 41.2 & 53.6 & 74.4 & 100.5 & 112.2 & 107.2 & means \\
\hline & 3.7 & 13.9 & 20.4 & 26.9 & 36.5 & 30.5 & sem \\
\hline
\end{tabular}

The represented values are the means \pm standard error of the means from of the thrombus parameters, expressed as percentages of the 3 control thrombi, induced before ASA superfusion is started.

Thrombosis is induced by ADP $\left(4 \times 10^{-4} \mathrm{M}\right)$ for 45 seconds; ASA $\left(10^{-2} \mathrm{M}\right)$ is added to the superfusion mixtures after the registration of the third control thrombus, and continuously superfused over the arterial segment.

Number of experimental animals $=\mathbf{5}$.

Table 3 Effect of continuous ASA supersion on thromboformation induced by ADP in diabetic rats.

\begin{tabular}{|l|rrrrrr|l|}
\hline \multirow{2}{*}{ Parameter } & \multicolumn{7}{|c|}{ Superfusion (minutes) } \\
& \multicolumn{1}{|c}{10} & \multicolumn{1}{c|}{22} & 34 & \multicolumn{1}{c|}{46} & \multicolumn{1}{c|}{58} & \multicolumn{1}{c|}{70} & \\
\hline$m_{(T)}$ & 59.1 & 53.0 & 73.2 & 94.6 & 75.0 & 75.8 & means \\
& 4.5 & 8.0 & 17.2 & 8.2 & 17.3 & 10.3 & sem \\
$m(D)$ & 61.5 & 58.6 & 73.7 & 84.4 & 74.4 & 73.2 & means \\
$T V M$ & 7.3 & 8.0 & 13.1 & 6.8 & 8.1 & 9.4 & sem \\
& 55.3 & 49.7 & 81.3 & 80.7 & 81.6 & 88.8 & means \\
$T T V$ & 9.7 & 5.1 & 20.5 & 9.5 & 21.6 & 4.2 & sem \\
& 50.8 & 43.2 & 69.8 & 68.4 & 69.6 & 77.3 & means \\
& 11.6 & 6.5 & 17.7 & 8.0 & 22.5 & 16.1 & sem \\
\hline
\end{tabular}

The represented values are the means \pm standard error of the means of the thrombus parameters, expressed as percentages of the 3 control thrombi, induced before ASA superfusion is started.

Thrombosis is induced by ADP $\left(4 \times 10^{-4} \mathrm{M}\right)$ for 45 seconds; ASA $\left(10^{-2} \mathrm{M}\right)$ is added to the superfusion mixtures after the registration of the third control thrombus, and continuously superfused over the arterial segment.

Number of experimental animal $s=5$.

as revealed by previous investigations using transmission electron microscopy.

\section{Registration of the thrombus parameters}

The results are summarized in Tables 2,3 and 4 . In the normal as well as in the "old diabetic" rats, continuous ASA superfusion results in a transient decrease in thromboformation. In normal rats, minimal values are obtained after 10 minutes of drug superfusion while the thrombus parameters regain their normal values seventy minutes later. The results of the regression analysis for both groups are summarized in Table 5. In diabetic animals, the decrease is maximal within twenty-two minutes of 
Table 4 Effect of superfusion with tranylcypromine on thromboformation induced by ADP, in normal and diabetic rats.

\begin{tabular}{|l|ccc|l|}
\hline \multirow{2}{*}{ Parameter } & Normals & $\begin{array}{c}\text { During tpanylcypromine } \\
\text { Sign. }\end{array}$ & Diabetics & \\
\cline { 2 - 4 }$m_{(T)}$ & 2.862 & $\mathrm{p}<0.05$ & 2.426 & means \\
& 0.106 & & 0.823 & sem \\
$m_{(D)}$ & 2.513 & $\mathrm{p}<0.01$ & 1.959 & means \\
$T V M$ & 0.078 & & 0.109 & sem \\
& 1.543 & $\mathrm{p}<0.01$ & 0.832 & means \\
$T T V$ & 0.120 & & 0.010 & sem \\
& 1.722 & $\mathrm{p}<0.001$ & 1.092 & means \\
& 0.078 & & 0.075 & sem \\
\hline
\end{tabular}

The represented values are the means \pm standard error of the means of the logarithms of the thrombus parameters, under tranylcypromine superfusion.

Thrombosis is induced by ADP $\left(4 \times 10^{-4} \mathrm{M}\right)$ for 45 seconds; tranylcypromine is added to the superfusion mixtures 2 minutes before thrombus induction.

Number of experimental animals in the normal as well as in the diabetic group $=5$.

Table 5 Effect of continuous ASA superfusion in normal and diabetic rats.

\begin{tabular}{|c|rrrrr|l|}
\hline \multirow{2}{*}{ Animals } & \multicolumn{7}{|c|}{ Superfusion (minutes) } & \\
& 0 & 12 & 24 & \multicolumn{1}{c|}{36} & 48 & \\
\hline \multirow{2}{*}{ Normals } & 46.7 & 58.8 & 75.5 & 101.7 & 103.1 & means \\
& 3.8 & 7.9 & 9.6 & 14.6 & 14.6 & sem \\
\hline \multirow{2}{*}{ Diabetics } & 51.1 & 74.5 & 82.0 & 75.2 & 78.8 & means \\
& 3.5 & 8.0 & 4.3 & 8.5 & 5.5 & sem \\
\hline
\end{tabular}

The represented values are the means \pm standard errors of the means of all thrombus parameters, beginning with the minimal values and expressed as percentages of the control thrombi.

For normals:

Regression equation: $\mathrm{y}=77.16+1.30(\mathrm{x}-24.00)$

Correlation value: $\mathrm{r}^{*}=0.9878$

For diabetics:

Regression equation: $\mathrm{y}=72.32+0.47(\mathrm{x}-24.00)$

Correlation value: $\mathrm{r}^{*}=0.8108$

Slope difference: $t=2.7181 \quad($ d.f. $=6) \quad \leq 0.05$

ASA superfusion, and values have not yet normalized at the end of the registration period. Tranylcypromine induces an increase in thromboformation to a larger extend in normal than in "old diabetic" rats.

\section{Discussion}

Diabetes is considered to be a main risk factor for atherosclerosis and it has been proposed that this disease could accelerate the atherosclerotic process through a mechanism involving injury of the endothelial cells. In contrast to the early manifestations of diabetes, characterized by well defined biochemical alterations ${ }^{11}$, the vascular abnormalities become only manifest in the later stages of the disease, the morphological changes depend largely on the nature of the artery. 
In this investigation, our aim was to study the involvement of the arachidonate cascade in arterial thrombosis in diabetic animals as compared to normal ones. The loss in weight of the diabetic rats and the increase in vessel wall thickness (Table 1), together with the manifest changes in concentrations of glucose, phospholipids and insulin are in full agreement with the results published previously by our group ${ }^{17)}$ and others ${ }^{18,19)}$. The dilated state of the arteries was already described by Bohlen and Hankins ${ }^{20}$ in intestinal arterioles, but the unexpected finding is that control thrombi, registered from "old diabetics" are definitely smaller than those from control rats. The mean value of $m_{(T)}$ for instance of all control thrombi of normals equals $519 \mathrm{mV}$, reaching only $256 \mathrm{mV}$ in the diabetic rats. Intriguingly, although the arteries of "young diabetics" are equally dilated, the registered thrombi do not differ very much from those of untreated animals (results not shown). As thrombus growth is amongst other factors related to the dimension and the severity of the induced lesion, it was deemed essential to determine the reaction of the vessel wall to the applied electrical current in the three groups.

SEM reveals that only the lesion in the "young diabetic" group is larger than in the normal control group (Fig. 2, 3 and 4), which is further confirmed by planimetric estimations of the surfaces of the different lesions, determined on SEM pictures taken at identical magnifications. The size of the de-endothelialized area is comparable in normal and in "old diabetic" animals, although a higher current is administered to the arteries of the latter group, related to the larger diameter of the vessels. Conversely, although the diameters of the vessels are equally increased, the desquamated area's are definitely larger in "young diabetic" rats. On the contrary, the wall thickness of the "old diabetic" arteries exceeds markedly the thickness of the normal vessels, and even more that of the "young diabetic" group. This latter difference could well explain the marked increase in size of the desquamation in the "young diabetic" animals. Evidently, the projected arterial segment on the light detecting elements of the optoelectronic registration device is identical in dimension independently from a normal or a dilated state of the artery; this results in an apparently smaller thrombus size in the dilated arteries of the "old diabetic" group.

Previous studies gave evidence of the role of the prostaglandin biochemical pathway in platelet-vessel wall interaction and local arterial thrombosis ${ }^{13)}$. Topical superfusion over the arterial segment by prostacyclin $\left(\mathrm{PGI}_{2}\right)$ resulted in a dose dependent reduction in thromboformation, whereas the application of tranylcypromine, an inhibitor of prostacyclin synthetase, induced a substantial increase of the thrombus parameters. The transient decrease in thrombosis, caused by continuous cyclooxygenase inhibition through ASA superfusion, can be explained by changes in the ratio of proaggregating cyclic endoperoxides versus the antiaggregating $\mathrm{PGI}_{2}$.

When the cyclooxygenase activity of the arterial vessel wall is inhibited, the diabetic specimens respond in a way quite similar to normal specimens. Inhibition, although of the same magnitude, occurs twelve minutes later in our system and normalization is not yet observed after seventy-two minutes. Several mechanisms could account for this difference in vascular reaction 
pattern between normal and diabetic animals: possibly a decrease in cyclooxygenase activity, or less access to the enzyme itself or eventually a retarded release of the cyclooxygenase-dependent metabolites. As superfusion by ASA equally reduces thromboformation in both groups, the possibility of less enzymatic activity can be excluded; furthermore, sustained inhibition of $\mathrm{PGI}_{2}$ synthetase by tranylcypromine does not induce more (retarded) enhancement of thromboformation, excluding as such the possibility of a retarded release of proaggregating prostanoids. As far as the cyclooxygenase activity of the arterial wall is considered, the remaining hypothesis is a decrease of the availability of the enzyme possibly through a mechanism of less access to this moiety. Additionally, by comparing the results observed when $\mathrm{PGI}_{2}$ synthetase is inhibited in normal and diabetic rats, less increase in thromboformation could well be related to a decrease in the activity of the enzyme itself.

In conclusion, this in vivo study reveals that a reduction in vascular $\mathrm{PGI}_{2}$ synthetase and not cyclooxygenase activity, accounts for the diminished release of prostacyclin from arteries of streptozotocin induced diabetic rats. Electrically induced de-endothelialized areas are larger in young diabetic animals as the increase in blood vessel diameter is not yet accompanied by smooth muscle cell proliferation. The size of the thrombus induced in dilated hypertrophic arteries of old diabetic rats appears to be smaller when compared to control thrombi in normal arteries but this apparent discrepancy in magnitude is due to the difference in scale of the microprojection performed by the optoelectronic device.

\section{References}

1) Sherwin, R., Felig, P.: Pathophysiology of diabetes mellitus. Med. Clin. North Am., 62; $695 \sim 711,1978$.

2) Kilo, C., Vogler, N., Williamson, J.R.: Muscle cappilary basement membrane changes related to ageing and to diabetes mellitus. Diabetes, 21; 881 901, 1972.

3) Ross, R., Glomset, J.A.: The pathogenesis of atherosclerosis. N. Engl. J. Med., 295; 369 377, 1976.

4) Bierman, E.L., Brunzell, J.D.: Interrelation of atheroselerosis, abnormal lipid metabolism and diabetes mellitus, in: Advances in Modern Nutrition vol. 2 (Katzen, H., Mahler, R., eds), Hemisphere Publishing Corporation, Washington: 187 210, 1978.

5) Halushka, P.V., Lurie, D., Calwell, J.A.: Increased synthesis of prostaglandin E-like material by platelets from patients with diabetes mellitus. N. Engl. J. Med., 297; 1306 1310, 1977.

6) Silberbauer, K., Schernthaner, G., Sinzinger, H., Pizakatzer, H., Winter, M.: Decreased vascular prostacyclin in juvenile onset diabetes. N. Engl. J. Med., 300; 366 367, 1979.

7) Harrison, H.E., Johnson, M.: Vascular prostacyclin release and metabolic dearrangement in diabetes. Horm. Metab. Res. suppl. 11; 43 49, 1981.

8) Davis, T.M., Mitchell, M.D., Turner, R.C.: Circulating prostacyclin may be reduced in diabetes, Lancet, 2; 789, 1979.

9) Harrison, H.E., Reece, A.H., Johnson, M.: Decreased vascular prostacyclin in experimental diabetes. Life Sci., 23; 351 356, 1978.

10) Rosen, P., Schrör, P.: Increased prostacyclin release from perfused hearts of acutely diabetic rats. Diabetologia, 18; 391 394, 1980.

11) Sterin-Borda, L., Gimeno, M., Borda, E., del Castillo, E., Gimeno, A.L.: Prostacyclin 
$\left(\mathrm{PGI}_{2}\right)$ and $\mathrm{U}-46619$ stimulate coronary arteries from diabetic dogs and their action is influenced by inhibitors of prostaglandin synthesis. Prostaglandins, 22; 267 278, 1981.

12) Bourgain, R.H., Vermariën, H., Andries, R., Vereecke, F., Jacqueloot, J., Rennies, J., Blockeel, E., Six, F.: A standardized i.l vivo method for the study of experimental arterial thrombosis: description of a meshod, in: Oxygen Transport to Tissue vol. 6 (Bruley D., Bicher H., Reneau D., eds), Plenum Publishing Corporation, New York: 635 649, 1985.

13) Bourgain, R.H., Andries, R., Maes, L.: Effect of cyclooxygenase inhibition on platelet-vessel wall interaction. Haemostasis, 13; 102 108, 1983.

14) Potvliege, P., Bourgain, R.H.: Thrombosis induced in the mesenteric artery of rats. An electron microscopic study of the initial phases. Br. J. Exp. Pathol., 57; 722 732, 1976.

15) Maes, L., Andries, R., Bourgain, R.H.: Endothelial injury and platelet thrombosis in mesenteric arteries of rats. A scanning microscopy study. Blood Vessels, accepted for publication.

16) Reidy, M.A., Schwartz, S.M.: A technique to investigate surface morphology and endothelial cell replication of small arteries: a study in acute angiotensin induced hypertensive rats. Microvasc. Res., 24; 158 167, 1982.

17) Wu, J.X., Maes, L., Andries, R., Warson, F., Gepts, W., Bourgain, R.H.: Early morphologic changes in coronary arteries of experimental diabetic rats. Acta Diabetol. Lat., accepted for publication.

18) Reinilä, A.: Long-term effects of untreated diabetes on the arterial wall in rats, Diabetologia, 20; 205 212, 1981.

19) Wilson, R.B., Martin, J., Hartroft, W.: Evaluation of the relative pathologic roles of diabetes and serum cholesterol levels in the development of cardiovascular lesions in rats. Diabetes., 16; 71 82, 1967.

20) Bohlen, M.G., Hankins, K.D.: Early arteriolar and capillary changes in streptozotocin induced diabetic rats and intraperitoneal hyperglycaemic rats. Diabetologia, 22; 344 $348,1982$.

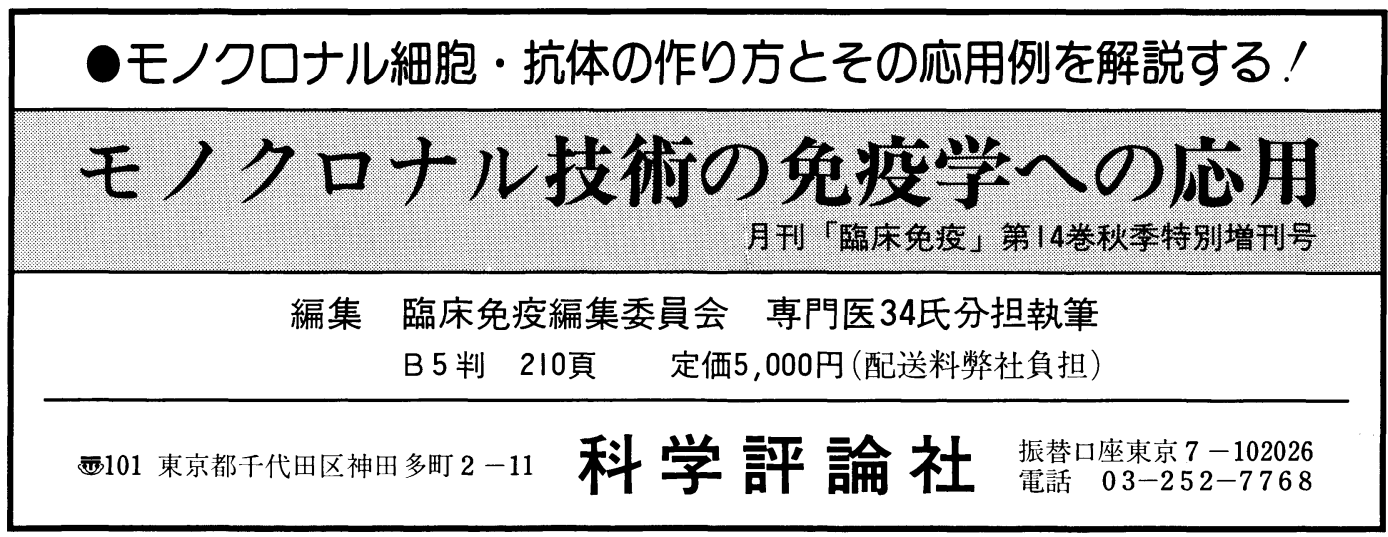

